\title{
[gw22-e0881] NORMAL CORONARY ANGIOGRAPHY PATIENTS WITH COLLATERAL CIRCULATION FROM RIGHT CORONARY ARTERY TO LEFT IN AORTIC VALVULAR STENOSIS: ONE CASE
}

Xie Dongyang, Liu Hairong, Xie Dongming Department of Cardiology, Affiliated Hospital of Gannan Medical College, JiangXi Ganzhaou, China

10.1136/heartjnl-2011-300867.497

Case Study Clinical Data Patient: Male, 52 years old, because of exertional chest tightness, dyspnea and fatigue for 3 years, aggravated half a months, admitted to hospital on August 28, 2010: blood pressure $120 / 75 \mathrm{~mm} \mathrm{Hg}$, the apex beat was located at outside $2 \mathrm{~cm}$ of left space midclavicular line, in the sixth intercostal space, right sternal border of the second intercostal space had thrill, heart border was to bottom left expansion. The heart rate was $112 \mathrm{bpm}$, cardiac tone of irregular force, and heart rhythm was absolutely irregular. There had IV/6 systolic murmur at right sternal border of the second intercostal space, conducted to the neck, mezzo diastolic murmur could be heard at apex of heart. By echocardiography tips: rheumatic heart disease with aortic stenosis (moderate), mitral valve stenosis (moderate), left atrioventricular enlargement. Left ventricular had diastolic dysfunction, but systolic function was normal. This patient did coronary angiography before surgical valve replacement. The coronary angiography results: both mitral valve and aortic valve were significant calcification. Left coronary artery angiography tips: the left main artery, left anterior descending (LAD) artery and circumflex branch artery were normal distribution, and no calcification and stenosis, blood flow was TIMI 3 level (figure 1 and figure 2), left main blood pressure was 95/65 $\mathrm{mm} \mathrm{Hg}$ (mean $81 \mathrm{~mm} \mathrm{Hg}$ ). The right coronary angiography Tips: the right main artery and branches were normal distribution, and no calcification and stenosis, blood flow was TIMI 3 level (figure 3), right coronary artery opening blood pressure was 130/80 (mean $102 \mathrm{~mm} \mathrm{Hg}$ ). But we could see collateral circulation from the distal segment of right coronary artery ahead to distal segment of LAD, the distal artery of LAD and its branches were clearly developed, up to rantrop II level flow. Analysed the data of the left coronary artery angiography again, both left main coronary artery and LAD had no stenosis in proximal and middle artery. 